\title{
LA DETERMINACIÓN MULTIDIMENSIONAL DEL DESEMPEÑO EMPRESARIAL SOSTENIBLE
}

\author{
Dra. Vilnia Elena Galán Rivas \\ Centro Universitario Mayarí. Universidad de Holguín. Cuba. \\ Dra. Milagros Morales Pérez \\ Universidad de Oriente. Cuba \\ Contacto: vgalanr@cum.uho.edu.cu \\ milagros@uo.edu.cu
}

Fecha de recepción: 19/10/2015

Fecha de aceptación: 23/12/2015

\begin{abstract}
Resumen
En la medida en que el entorno empresarial se ha modificado el desempeño de las entidades ha cobrado diversos matices, exigiendo un enfoque diferente. El presente artículo tiene como objetivo analizar la determinación multidimensional del Desempeño Empresarial Sostenible. El análisis crítico de la bibliografía especializada centró la atención en los objetivos que persiguen cada propuesta, las dimensiones y los indicadores que se evalúan.

Se demuestra: que las propuestas analizadas son, mayoritariamente, unilaterales y excluyentes, con un sesgo a favor del aspecto económico-financiero y la supresión preceptiva de la dimensión medioambiental y social; la necesidad del enfoque multidimensional del desempeño empresarial sostenible para lograr un análisis más integral de la relación de la empresa con su entorno; que sus cuatro dimensiones (ambiental, económico -tecnológica, legal, y social), representan sus determinaciones cualitativas, que posibilitan un enfoque multilateral y permiten identificar las determinaciones cuantitativas para su medición y evaluación.
\end{abstract}

Palabras claves: Desempeño Empresarial Sostenible, determinación multidimensional.

THE MULTI-DIMENSIONAL DETERMINATION OF SUSTAINABLE BUSINESS PERFORMANCE

Summary

Since the business environment has modified the performance of the entities it has charged diverse nuances, demanding a different focus. The present article has as objective to analyze the multidimensional determination of the Sustainable Business Performance. The critical analysis of the specialized bibliography centered the attention in the objectives that pursue each proposal, the dimensions and the indicators that are evaluated.

It is demonstrated: that the analyzed proposals are, for the most part, unilateral and excluding, with a bias in favor of the economic-financial aspect and the mandatory suppression of the environmental and social dimension; the necessity of the multidimensional focus of the sustainable business performance to achieve a more integral analysis of the relationship of the 
company with their environment; that its four dimensions (environmental, economic technological, legal, and social), represent their qualitative determinations that facilitate a multilateral focus and they allow to identify the quantitative determinations for their measuring and evaluation

Key words: Sustainable Business Performance, multidimensional determination

\section{INTRODUCCIÓN}

La inclusión de los problemas medioambientales en el objeto de estudio de la economía y en particular de la economía aplicada, constituye un extenso capítulo en la historia de la ciencia. La economía al estudiar, en sentido general, la asignación eficiente de recursos limitados o escasos, presenta la eficiencia en el sentido de Pareto ${ }^{1}$ como paradigma de la racionalidad económica. Sin embargo, la constatación de "fallas en los mercados" a partir de la aparición de externalidades, planteó ante los economistas un nuevo dilema según el cual, el comportamiento de unos agentes afectaba a otros sin que los costos inducidos se reflejaran en el sistema de precios. Los estudios realizados por A. C Pigou y posteriormente por R. H. Coase, se apoyaron en ejemplos de externalidades negativas por contaminación y trajeron al centro del debate económico la cuestión de los costos privados frente a los costos sociales. La realidad rebasaba el principio de optimalidad de Pareto y esta nueva contradicción ampliaba el campo de investigación de la ciencia económica.

La agudización de los problemas medioambientales y la visión más consciente y consensuada de la complejidad de los mismos, ha coadyuvado al estudio y la incorporación de la "sostenibilidad" como un elemento de la gestión empresarial. Este cambio de actitud es consecuencia de las transformaciones institucionales y el desarrollo de iniciativas a nivel local, regional e internacional.

En la medida en que el entorno empresarial se ha modificado el desempeño de las entidades ha cobrado diversos matices en el orden conceptual y práctico, definiéndose no solo desde un enfoque económico.

A finales del siglo pasado se desarrollaron esfuerzos para construir un marco regulatorio que propiciara la incorporación de la dimensión medioambiental al desempeño empresarial. En 1996, auspiciado por la oficina internacional de normalización (ISO) entró en vigor la ISO 14001 referida a la gestión ambiental y más tarde en 1999 la ISO 14031, con el objetivo de reglamentar la evaluación del desempeño ambiental de una organización. Posteriormente aparece la dimensión social con la introducción de los términos desempeño socialmente responsable y responsabilidad social empresarial.

No obstante la conceptualización del desempeño empresarial como un proceso multidimensional, es extremadamente limitada en la bibliografía especializada y en los documentos programáticos de organizaciones internacionales y nacionales, que se emiten

\footnotetext{
${ }^{1}$ La eficiencia económica en el sentido de Pareto se define como aquella situación en la cual no es posible mejorar el bienestar de una persona sin empeorar el de alguna otra.
} 
con el propósito de mejorar el marco regulatorio de la actividad empresarial con relación al medioambiente.

La presente investigación estudia el tránsito objetivo del concepto de desempeño empresarial hacia una nueva definición categorial: el Desempeño Empresarial Sostenible (DES). Este proceso supone la incorporación de nuevas variables, hasta alcanzar un enfoque multidimensional del desempeño empresarial, que supere la concepción tradicional que, de manera unilateral, lo define con un sesgo a favor de la dimensión económica, asociándolo directamente con términos como eficiencia, excelencia y competitividad.

Por tanto se define como su objetivo: Analizar la determinación multidimensional del desempeño empresarial sostenible a través del estudio crítico de la bibliografía especializada.

\section{DESARROLLO}

La aplicación de un modelo insostenible que no tiene en cuenta su incompatibilidad con el ritmo de regeneración de los recursos naturales renovables, la imposibilidad de reposición de los recursos no renovables, la emisión creciente de residuos y la capacidad de carga de los ecosistemas, exige soluciones rápidas y eficaces que permitan conciliar intereses políticos, económicos, sociales, jurídicos y ambientales, requiriendo un desempeño responsable de los agentes económicos y sociales. La conceptualización del DES desde un enfoque multidimensional es una necesidad perentoria de la ciencia. Dado que el pensamiento científico solo reproduce la realidad a través de conceptos ${ }^{2}$, resulta imprescindible, en el marco de cualquier investigación, establecer las dimensiones cualitativas del objeto que hacen posible su definición categorial. De esta manera se sientan las bases para el estudio de las influencias recíprocas sobre las partes que lo componen, su medición, el pronóstico de su posible desarrollo ulterior y la elaboración de recomendaciones dirigidas a transformar o mitigar, el impacto de factores indeseables (externalidades negativas).

La cultura empresarial, como elemento integrante del valioso legado de la humanidad, debe orientarse a la exigencia desde adentro, para alcanzar economías de escala creciente en materia de beneficios, pero también del entorno que se nutre y forma parte. Por ello, la relación empresa-medio ambiente debe basarse en la premisa inviolable de que el desempeño empresarial debe ser estrictamente sostenible. Sin embargo, este objetivo no se alcanzará sin la formación de nuevos valores en los "clientes internos", una verdadera actitud práctico-crítica ante la realidad medioambiental circundante.

\footnotetext{
${ }^{2}$ Marx señala que el pensamiento científico, con ayuda de las categorías y conceptos, reproduce la realidad histórica objetiva como lo concreto-pensado, como la "síntesis de múltiples determinaciones". Ver: Marx, Karl (1971), "Fundamentos de la Crítica a la Economía Política”.
} 


\subsection{El Desempeño Empresarial en la teoría e historia económica: un análisis crítico.}

El análisis crítico representa la forma en que el pensamiento científico "reconstruye" idealmente la realidad, a partir de nuevos conceptos. A la ciencia le es inmanente una mirada crítica sobre el objeto que se investiga; la crítica teórica expresa la continua reflexión del pensamiento científico, la mediación que este realiza entre la realidad y el concepto, entre la realidad perceptible y el "estado de las artes"; por consiguiente la crítica teórica es indispensable para el desarrollo de la ciencia.

El análisis crítico del desempeño empresarial permite observar la evolución de este concepto en el tiempo. Desempeño es un vocablo tomado del inglés y relacionado con el logro de objetivos, de ahí que cuando se habla de desempeño empresarial en el orden más general, se entiende como el cumplimiento cabal de las obligaciones establecidas por los sujetos económico-sociales. Esto explica que se asocie con los términos eficiencia, excelencia y competitividad, pues la razón de ser de una empresa, es la producción eficiente de bienes y servicios.

En la década de los 60 Peter Drucker, exponía sus ideas acerca del desempeño empresarial, planteando que la eficiencia significa hacer correctamente las cosas mientras que eficacia es hacer las cosas correctas (Drucker, 1964).

La eficiencia, o sea la capacidad de hacer correctamente las cosas, es un concepto de "entrada-salida" (insumo-producto). El administrador eficiente es aquel que logra las salidas, o resultados, que corresponden a las entradas (mano de obra, materiales y tiempo) utilizadas para conseguirlos. Los que logran minimizar el costo de los recursos con que obtienen sus metas están obrando de manera eficiente (Stoner, 1997).

Si la empresa logra producir con eficiencia y eficacia será una entidad de excelencia y competitiva, entendido esto último como..."la capacidad de una empresa u organización de cualquier tipo para desarrollar y mantener unas ventajas comparativas que le permiten disfrutar y sostener una posición destacada en el entorno socio económico en que actúan. Se entiende por ventaja comparativa aquella habilidad, recurso, conocimiento, atributos, etc., de que dispone una empresa, de la que carecen sus competidores y que hace posible la obtención de unos rendimientos superiores a estos" (Bueno, 1989) Significa, que en el orden económico una empresa debe, en primer lugar, lograr eficiencia, eficacia y competitividad $^{3}$.

\footnotetext{
${ }^{3}$ Es Michael Porter quién desarrolla el concepto de competitividad en sus obras "Estrategia Competitiva" (1980), "Ventaja Competitiva" (1985) y "Las Ventajas Competitivas de las Naciones" (1990), las que despertaron el interés de académicos, políticos y empresarios. En general "... se entiende por competitividad la capacidad que tiene una firma de satisfacer una necesidad revelada en el mercado por parte de los consumidores, de una forma más eficiente en comparación con otra firma que también posee capacidad de satisfacer la misma necesidad. Lo anterior tiene relación con las necesidades presentes; sin embargo, es importante tener en cuenta, que la competitividad existirá en la medida que la firma sea capaz de adelantarse a una necesidad futura... la competitividad es cómo se ganan partes del mercado, no necesariamente a costa de otros, ya que el mercado se puede ampliar". ver: Castell, Manuel (2008) ".Globalización, tecnología, trabajo, empleo y empresa", Revista "La Factoría" No. 7, octubre, España, www.lafactoriaweb.com.
} 
Sin embargo, la inclusión del problema del daño inducido o externalidades negativas, como una consecuencia de la acción de las empresas y la solución eficiente del mismo, es un tema antológico en la historia de la ciencia económica. Las empresas eficientes y competitivas pueden perjudicar a otras empresas o a comunidades aledañas. El asunto fue desarrollado por A. C. Pigou en su trabajo "The Economics of Welfare" (1932), en el cual le asigna un papel determinante al estado, como corrector de las fallas del mercado y factor determinante en la consecución del bienestar social. Posteriormente, Ronal H. Coase se encargaría de significar el hecho de que: "... la regulación gubernamental directa no necesariamente presentará mejores resultados que la solución de mercado o a través de la empresa. Pero igualmente, no hay razón por la que, en ocasiones, tal regulación administrativa gubernamental no deba conducir a un aumento de la eficiencia económica. Esto parecería particularmente probable cuando, como es normalmente el caso con la molestia por el humo, un gran número de personas son afectadas y en el que, por lo tanto, los costos de manejar el problema a través del mercado o la empresa pueden ser altos". (Coase, 1960).

Sin embargo, el problema de los costos sociales sigue siendo hoy un tema de intensas discusiones. La elevación de la producción, la provisión de fuentes de empleo y el mejoramiento del bienestar material en general puede ciertamente, en algunos casos, comprometer el medio ambiente. ¿Cuándo se puede decidir a favor de una u otro? Este problema se hace más complejo por el hecho de que las agresiones al medio ambiente tienen efecto en el largo plazo, mientras el bienestar se aprecia inmediatamente. Pero lo que en su momento significó Coase fue que las soluciones que no venían por la vía del mercado, también tenían costos implícitos: "Todas las soluciones tienen costos y no hay razón para suponer que la regulación del Gobierno será beneficiosa, aunque el problema no esté bien manejado por el mercado o por la empresa. Visiones satisfactorias de política sólo pueden provenir de un estudio consciente de cómo el mercado, las empresas y el Gobierno manejan, en la práctica, el problema de los efectos nocivo". (Coase, 1960).

Es precisamente, la visión más consciente y consensuada de la complejidad de los problemas medioambientales y las vías para solucionarlos, lo que ha posibilitado la inclusión paulatina de la "sostenibilidad" como un elemento de la gestión empresarial. Esta inclusión es consecuencia de los cambios institucionales y el desarrollo de iniciativas a nivel local, regional e internacional.

En la medida en que el entorno empresarial se ha modificado, el desempeño de las entidades ha cobrado diversos matices en el orden conceptual y en la medición, definiéndose no sólo desde un enfoque económico sino incorporando variables ambientales y sociales, que le otorgan un enfoque multidimensional. Ello ha sido una consecuencia lógica de la necesidad imperiosa de que la empresa pueda jugar su papel como agente económico del desarrollo local sostenible, a través de la sostenibilidad en su desempeño.

A finales de la década de los 80 comienza a modificarse el enfoque del desempeño, a partir de los hechos siguientes:

- En 1987, se publica el informe "Nuestro futuro común", por la Comisión Mundial sobre Medio Ambiente y Desarrollo de Naciones Unidas (CNUMAD), en el que se 
ofrece una visión crítica del modelo de desarrollo económico que hasta ese entonces había prevalecido y que se asumía por el sector empresarial en detrimento de los recursos naturales y se da una definición de desarrollo sostenible.

- En 1989 el Programa de las Naciones Unidas para el Medio Ambiente (PNUMA), propone la producción más limpia (PML), la cual enfatiza en la importancia de la gestión y organización efectiva de las empresas y la necesidad de la mejora continua en su desempeño ambiental.

- En 1991 se celebra la Segunda Conferencia Mundial de la Industria sobre la Gestión del Medio Ambiente, la Cámara Internacional de Comercio presentó la "Carta de las empresas para un desarrollo sostenible", la cual estaba respaldada por 500 empresas de todo el mundo y las comprometía a "mejorar su rendimiento ambiental".

- En 1996, auspiciado por la Organización Internacional de Normalización (ISO), entra en vigor las Normas ISO 14001 y 14004 acerca de la gestión la ambiental, y se comienza a hablar con fuerza del desempeño ambiental y en 1999 entra en vigencia la norma ISO 14031, con el objetivo de reglamentar la evaluación del mismo en una organización, convirtiéndose por tanto en un nuevo procedimiento de medición del desempeño.

- En el año 2002 fue aprobado por las Naciones Unidas el Pacto Mundial, normativa voluntaria de nueve principios concernientes a los derechos humanos, trabajo y medio ambiente.

- En el año 2008 aplicación de la norma 26. 000 para regular la responsabilidad social en las organizaciones.

Se puede apreciar que a finales de los años 80 comienza a gestarse un movimiento internacional que transforma paulatinamente, el marco institucional de actuación de las empresas. Este proceso incentiva la adopción de posturas amigables con respecto al medioambiente. La actitud de las empresas responde al hecho de que la nueva institucionalidad, es respaldada por millones de personas en el mundo que representan clientes potenciales, dispuestos a consumir productos generados por empresas, que asuman la sostenibilidad dentro de su gestión.

Concretamente, el marco institucional regulatorio que se promueve a finales del siglo XX, produce cambios en la gestión empresarial en las direcciones siguientes: adoptar métodos de producción ecoeficientes para mejorar tanto la eficiencia operativa como el posicionamiento en el mercado; la reducción de costos a causa del incremento de la eficiencia y eficacia operativa; el crecimiento en los ingresos de la empresa, debido a la diferenciación en sus productos y al aumento de su aceptación en el mercado; la mejora en la utilización de los recursos humanos y materiales.

El desempeño empresarial compatible con el principio de sostenibilidad, se destaca por erigirse sobre tres pilares fundamentales: el crecimiento económico, el balance ecológico y el progreso social. Los mismos son incluidos, de una forma u otra, en las propuestas de medición del desempeño empresarial.

En la investigación fueron objeto de revisión los procedimientos metodológicos siguientes: el Cuadro de Mando Integral (CMI); medición del desempeño basado en indicadores no financieros; medición del desempeño considerando la cadena valor, 
medición del desempeño de los recursos humanos, Norma ISO 14031, la norma de aseguramiento AA 1000-ACCOUNTABILITY, evaluación de la sostenibilidad corporativa (ESC) y desempeño socialmente responsable, la metodología para la evaluación del desempeño empresarial a partir de la organización de los procesos y el procedimiento metodológico para el análisis de la eficacia y eficiencia de un sistema empresarial.

En el análisis de las distintas propuestas centra la atención en los siguientes momentos: los objetivos que se persiguen, las dimensiones que se evalúan y los indicadores utilizados a tales efectos, llegando a los siguientes resultados:

a) Las principales formulaciones del CMI, aparecieron a principios de los años noventa y se deben a la labor de los autores (Kaplan y Norton 1997), quienes trabajaron en la medición del desempeño a través del proyecto denominado " La Medición del Resultado en la Organización del Futuro", que originó el desarrollo del Balanced Scorecard como un sistema integral de indicadores que permite desplegar tanto los grandes propósitos, como comunicar las estrategias y también alinear los distintos niveles y áreas con las estrategias; promoviendo el aprendizaje organizacional.

El cuadro de mando integral es una herramienta muy útil para el control de la gestión de las empresas y sus diversas áreas. En el CMI la intención es medir el progreso actual de la empresa y suministrar su orientación futura, y así convertir la visión en acción, mediante un conjunto coherente de perspectivas, objetivos, indicadores e iniciativas económicas. La medición es estrictamente económica-financiera y así lo corroboran los indicadores utilizados, que son: en el orden financiero la utilidad financiera, la siniestralidad y la utilidad técnica. Referido al cliente mediante el tiempo de suscripción y emisión, la encuesta de satisfacción y el porcentaje de participación mercado -poliza multiproducto. En lo interno, a través de la atención de requerimientos, colocación de negocios vs cotizaciones, por ciento de incremento de portafolios reaseguradores. El aprendizaje realizando encuesta de clima y cultura, con la evaluación semestral a funcionarios y el nivel de competencias.

Es decir, el CMI debe ser utilizado como un sistema de comunicación, información y formación más que como un sistema de control. Identificando fundamentalmente la perspectiva financiera del cliente interno, su formación y crecimiento. Permitiendo esto un equilibrio entre los objetivos a corto y largo plazo, con una clara unidad de propósito, la consecución de una estrategia integrada donde los sistemas de información deben comunicar y articular la estrategia empresarial.

b) La La medición del desempeño considerando la cadena valor (Moseng y Bredrup, 1993), es el segundo procedimiento analizado, el mismo consiste en un sistema organizado en diferentes niveles y grupos de indicadores, con el fin de presentar una visión jerárquica, coherente e íntegra de los procesos. El sistema de medición se estructura según los autores en tres niveles: el primer nivel considera el conjunto de la cadena de suministros y las interrelaciones entre las diversas actividades críticas; el segundo se centra en los principales procesos de la cadena, analizándose los procesos de gestión física de los 
productos, los procesos de compra y de negociación, y las unidades de stock en planta y el que tercer y último nivel controla cada actividad particular.

Los indicadores más utilizados para la evaluación en el orden económico-financiero son: los aspectos financieros tales como: presupuesto de la cadena de suministros, gasto personal, otros gastos, volumen de compra de productos en compra directa, inventario físico; la satisfacción del cliente, el cual comprende: período de respuesta a un pedido de reaprovisionamiento, número de productos-día en ruptura de inventario, índice de satisfacción del cliente; y los aspectos internos del proceso incluye: el índice de rotación del inventario, el número de horas trabajadas y el valor medio de una orden de compra. Nótese que estos dos procedimientos tienen en común que se mide el desempeño desde lo económico - financiero, y coinciden en que evalúan los aspectos financieros, de satisfacción del cliente externo e interno del proceso.

La diferencia está en el segundo caso, en que la medición se realiza atendiendo a diferentes niveles y el primero, incorpora la cuestión del aprendizaje y la medición. Es incuestionable la necesidad e importancia del análisis financiero empresarial, pero el desempeño de una organización no solo depende de los indicadores financieros, pues la calidad de los servicios y la satisfacción de los clientes constituyen factores que determinan el éxito o fracaso de una empresa.

c) La medición del desempeño basado en indicadores no financieros (Perera, 1997), tiene como objetivo evaluar los factores intangibles sobre los que se sustenta las ventajas competitivas de la empresa (calidad, flexibilidad, innovación, cliente), tanto en el orden cuantitativo como cualitativo y considerando aspectos del producto, del proceso y del cliente. Al igual que el anterior el enfoque es desde la dimensión económica, pero excluye el lado financiero y los indicadores que utiliza son: indicadores no financieros del producto (devoluciones, quejas o reclamaciones); indicadores no financieros del proceso (productos nuevos lanzados al mercado, habilidades en el diseño y mejoras en el producto, desviaciones en recursos, tiempo de utilización de las máquinas); e indicadores no financieros del cliente (pedidos entregados a tiempo, clientes satisfechos y tiempo empleado en la entrega de productos).

Estos aspectos de medición constituyen la respuesta de la contabilidad de gestión a la demanda empresarial de sistemas de medición, se diferencian de los tradicionales, permitan gestionar algunas variables que hoy en día se consideran claves para la toma de decisiones estratégicas en la empresa (Kaplan, 1984). En general, la utilidad de los indicadores no financieros para gestionar estrategias basadas en la calidad ha sido demostrada en las investigaciones realizadas al respecto, justificándose su capacidad para mejorar continuamente los resultados de la calidad, y los resultados que derivan de gestionar una producción orientada hacia el cliente (Perera, 1997).

d) Medición del desempeño de Recursos Humanos. (Wertner y Davis, 1996).

El objetivo es medir el desenvolvimiento del individuo en el cargo y su potencial de desarrollo. En este caso se evalúa el rendimiento de la fuerza de trabajo como factor de la producción, desde la perspectiva económica. Su medición se realiza a través de los 
indicadores: productividad del trabajo, correlación salario medio/productividad, cumplimiento de las acciones de capacitación, índice del cumplimiento del personal capacitado, el nivel de accidentalidad y el índice de utilización del fondo de tiempo laboral, entre otros.

Autores como Stoner refieren que la evaluación del desempeño de los recursos humanos es un proceso permanente que ofrece información a los empleados sobre lo bien que están desempeñando su trabajo en la organización. Además, existen modelos como el de las cuatro C: competencia, compromiso, congruencia y costos efectivos para evaluar la eficacia de los recursos humanos.

Esta evaluación permite establecer el vínculo de la persona con el cargo que desempeña, determina si posee un adecuado entrenamiento, si está incentivado para desarrollar su labor, si mantiene buenas relaciones humanas con sus superiores. Todo lo cual reportará beneficios a la empresa y permitirá determinar el potencial humano en el corto, mediano y largo plazo.

Obsérvese que los cuatro procedimientos analizados, miden el desempeño empresarial desde un enfoque económico- financiero, sin considerar variables ambientales, ni sociales.

e) La Norma ISO 14031 (1999), tiene como objetivo normalizar la medición del desempeño ambiental de las entidades, a través de un proceso interno, comparando el cumplimiento de las obligaciones ambientales de la empresa en el pasado con el período actual, una vez que sean establecidos determinados criterios en el orden ambiental. La norma permite, de esta manera, la identificación de los aspectos ambientales y evaluar su significancia. La evaluación de los aspectos ambientales se lleva a cabo considerando la frecuencia con que ocurre el aspecto, la probabilidad de que ocurra el impacto y la gravedad del impacto producido. Los indicadores que utiliza son los siguientes: consumo de agua, vertido al agua, energía emitida, uso de energía, emisiones a la atmósfera, descarga al suelo, uso de materias primas y recursos materiales, residuos y subproductos generados. Para ello la norma establece que el nivel de significancia se defina a partir de las puntuaciones obtenidas para la frecuencia, probabilidad y la gravedad de un aspecto ambiental, es decir, todas las actividades de una organización que pueden interactuar con el medio ambiente. La norma ISO 14004 de 1996, define los criterios que establece la norma para evaluar la frecuencia, probabilidad y gravedad de los aspectos ambientales, y se calcula el producto de ellas para obtener su significancia. Al valor obtenido se le asigna el nivel de significancia correspondiente considerando que un aspecto ambiental significativo es aquel en el que la significancia $S>100$.

La norma ISO 14031 evalúa el desempeño estrictamente desde lo ambiental, sin proponer el cálculo de los niveles de desempeño ambiental o valores de referencia. No obstante, la misma constituye un paso de avance en la medición del cumplimiento de la obligación ambiental pues exige que la empresa, como agente económico del desarrollo local sostenible, gestione adecuadamente los recursos naturales que utiliza para producir bienes y servicios y de esta forma asegurar un desempeño sostenible. 
A partir de este momento se van incorporando nuevos enfoques de desempeño empresarial, con una perspectiva multidimensional, nos referimos específicamente a los Reportes de Sustentabilidad, la norma de aseguramiento AA 1000 - ACCOUNTABILITY y al desempeño socialmente responsable. Los mismos guardan estrecha relación con la sostenibilidad del desempeño existiendo diversos motivos para que una empresa adopte el mismo dentro de su política, de su marco de gestión y del desarrollo de sus actividades, buscando la mejora continua en su forma de operar, en sus productos y servicios.

f) La norma de aseguramiento AA 1000 - ACCOUNTABILITY 2003, estándar voluntario desarrollado por el "Institute of Social and Ethical Accountability". Es un estándar de aplicación general para evaluar, atestiguar y fortalecer la credibilidad y calidad del informe de sostenibilidad de una organización y de sus principales procesos, sistemas y competencias.

Examina de manera exhaustiva cómo la organización comprende sus propios resultados e impactos, y los puntos de vista de las partes interesadas. Evalúa cómo la organización informante responde a las demandas de las partes interesadas y, al hacerlo, interpreta la preparación del informe como parte de un continuo compromiso con ellas.

La propuesta tiene como objetivo evaluar y fortalecer la credibilidad de los reportes del desempeño ético, económico y ambiental de las entidades. Los indicadores previstos son: cultura organizacional, comercio justo y ético, condiciones de trabajo, manejo del recurso humano y entrenamiento, protección ambiental y animal, desarrollo de la comunidad y derechos humanos. Debe resaltarse como positivo la perspectiva multidimensional de esta propuesta que no solo mide lo tradicionalmente económico, sino el desempeño ambiental e incluso lo ético, un momento importante de la conducta social de la entidad. No obstante, lo que se presenta es un reporte final cuyas características, como reporte al fin, no incluye cálculos y por tanto, no permite llegar a valores de desempeño.

g) La Evaluación de la Sostenibilidad Corporativa (ESC) (2000), es una propuesta del Instituto de Investigación Empresarial del Sustainability Asset Management Group, SAM, que tiene como objetivo evaluar el cumplimiento de las obligaciones ambientales, económicas y sociales de las entidades. Se considera necesario medir el desempeño atendiendo a las dimensiones: ambiental (política y manejo ambiental, desempeño ambiental- ecoeficiencia, información ambiental, desempeño ambiental avanzado, estrategia sobre cambio climático, generación de electricidad, transmisión y distribución, biodiversidad y proyectos de infraestructura); económica (Gobierno Corporativo, manejo de riesgos y crisis, códigos de conducta - cumplimiento -corrupción y soborno, manejo de relaciones con los clientes, administración de riesgos de precio, oportunidades de mercado, base de datos de clientes, manejo del riesgo en los precios); social (indicadores de prácticas laborales, desarrollo del capital humano, atracción y retención de talentos, salud ocupacional y seguridad, compromiso con las partes interesadas, ciudadanía corporativa y filantropía, información social). 
h) El desempeño socialmente responsable se relaciona directamente con la responsabilidad social empresarial, que se define, según World Business Council for Sustainable Development (WBCSD), Suiza como "el compromiso que asume una empresa para contribuir al desarrollo económico sostenible por medio de colaboración con sus empleados, sus familias, la comunidad local y la sociedad en pleno, con el objeto de mejorar la calidad de vida".

El mismo se relaciona con el desempeño sostenible empresarial integrando, los cinco elementos fundamentales son: comportamiento más ético, mejor clima laboral, mejor desempeño medioambiental, marketing más responsable y hacer una contribución al desarrollo social de la comunidad.

La medición del desempeño socialmente responsable propone medir la gestión de dicho desempeño y el desempeño propiamente dicho, En este último caso el enfoque, al igual que la Responsabilidad Social Corporativa es multidimensional pero explícitamente más amplio y se proponen evaluar las dimensiones: ética empresarial (desarrollo de principios éticos, difusión de los principios éticos de la empresa, práctica de los principios éticos con la competencia, práctica de los principios éticos con proveedores, práctica de los principios éticos con personal contratado, práctica de principios éticos con el gobierno); calidad de vida laboral (valorización y respeto de la diversidad, participación de los empleados en la gestión de la empresa, relación con grupos de trabajadores organizados y sindicatos, distribución de los beneficios de la empresa, desarrollo profesional y empleabilidad, cuidado de la salud, seguridad y condiciones de trabajo, jubilación y despidos); ambiental (impacto en el medio ambiente, responsabilidad ambiental a través del ciclo productivo, cuidados en la incorporación de insumos y tratamiento de desechos, educación ambiental); compromiso con la comunidad (gestión del impacto de las actividades de la empresa en la comunidad, relaciones con organizaciones comunitarias presentes en su entorno- ONG, colegios-, mecanismo de apoyo a proyectos sociales, estrategias y criterios de acción en inversiones sociales, apoyo al trabajo voluntario del personal, liderazgo e influencia social, participación en proyectos sociales del gobierno) y marketing responsable (diagnóstico y solución de los daños potenciales de sus productos y servicios, política de marketing y comunicación, técnicas de venta éticas y respeto de la privacidad del consumidor, excelencia en la atención al consumidor).

Esta propuesta en una misma dimensión mezcla cuestiones económicas y sociales, en el orden más general las dimensiones que se miden son la ambiental, económica y social. Independientemente de lo positivo de la propuesta en el orden de la explicites del enfoque multidimensional, la referencia a la educación ambiental es insuficiente y además a juicio de las autoras, no se formula el cálculo del desempeño.

i) Metodología para la evaluación del desempeño empresarial a partir de la organización de procesos, (Cardeñosa y Rodríguez 2002). Su objetivo es el de evaluar el desempeño empresarial. La misma consta de cuatro fases con sus correspondientes etapas, aspectos a considerar y resultados esperados. 
La primera fase es la caracterización general de la entidad objeto de estudio. Se inicia analizando como la organización interna del sistema responde a su Misión, la cual se definiría a partir de las exigencias político - económicas que ejerce el medio. Se determina si existen causas organizativas que están incidiendo en el funcionamiento interno del sistema y que afectan su correspondencia con el entorno.

La segunda fase es de diagnóstico, su objetivo es delimitar la naturaleza de los problemas organizativos que se detectan, a través del análisis de la eficiencia, haciendo uso de los resultados financieros y económicos del sistema para definir la naturaleza de los problemas organizativos y en qué medida están incidiendo en los resultados del sistema.

La tercera fase es de proyección de la solución, donde se abordará el análisis de los elementos de la organización de los procesos que se detecten como deficientes durante el diagnóstico. Se busca perfeccionar las formas y los métodos de organización, el aseguramiento de la cantidad, calidad y el aseguramiento del sistema de normas.

La última fase es la aplicación y es precisamente aquí, donde de definen las acciones a desarrollar para lograr el cambio organizativo, su ajuste y estabilización.

La metodología "Evaluación del desempeño empresarial a partir de la organización de los procesos", es bastante amplia y orgánica en la incorporación de las fases que la conforman, la misma se queda en el plano puramente económico al realizar un análisis de la situación económica -financiero de la empresa y solo profundiza en los aspectos relacionados con la organización de los procesos, a partir de la definición de indicadores técnicos organizativos, no tiene en cuenta la gestión de los recursos humanos, ni la variable ambiental, por tanto, no estamos en presencia de un análisis de sostenibilidad del desempeño empresarial, ni del cálculo del mismo.

j) Procedimiento metodológico para el análisis de la eficacia y eficiencia de un sistema empresarial (2005) y su aplicación con la tesis: Evaluación y mejora del desempeño empresarial a partir de los indicadores de eficacia y eficiencia en la línea de ventas minoristas de Artex Holguín S.A. En la misma se calcula el desempeño empresarial, midiendo la eficacia a través del indicador nivel de servicio, el cual es considerado en dos perspectivas, la del cliente externo, medida a través del índice de satisfacción del cliente y la del funcionamiento de los procesos internos del sistema medida de fiabilidad.

En un segundo momento se mide el índice de eficiencia empresarial teniendo en cuenta indicadores como rentabilidad económica, costo por peso de venta, rendimiento de activos fijos, productividad por trabajador y rotación de los inventarios. Aun cuando se reconoce la importancia y valides del procedimiento propuesto, a juicio de las autoras, son insuficientes los indicadores utilizados para evaluar el desempeño a partir de los indicadores de eficacia y eficiencia.

Se revisaron también otros procedimientos como el de: análisis y mejoramiento de procesos de Rodríguez Urquiaga ( 2003); el procedimiento para la gestión por procesos de Noqueira Rivera ( 2003),Tecnología para el proceso de cambio organizacional a través del enfoque de procesos de Hernández Concepción ( 2005); Procedimiento para medir la eficacia organizacional de Mayo Alegre( 2009), el objetivo de los mismos no es el de 
evaluar el desempeño, mayoritariamente se centran en el análisis de los procesos sin cálculos del desempeño empresarial y sin realizar un análisis de su sostenibilidad.

De las 14 propuestas metodológicas revisadas para medir el DES, a modo de resumen, se puede señalar lo siguiente:

- En 13 se evalúa la dimensión económica, para un 92,85\%.

- En el $50 \%$ se evalúa la dimensión ambiental y la social en el $25 \%$.

- En sólo dos (14,29\%), se habla de medición de desempeño utilizando el término sostenible.

- La dimensión legal no se mide en ninguna propuesta.

- En solo una de ellas (7,14\%), se incluyen cálculos para medir el desempeño.

Las propuestas analizadas tienen una gran importancia metodológica y práctica. Sin embargo, se observa que son, en casi todos los casos, unilaterales y excluyentes, con un sesgo a favor del aspecto económico financiero y la supresión preceptiva de la dimensión medioambiental y social. La otra insuficiencia notable es la carencia de un método de cálculo que permita medir formalmente, el desempeño. Por consiguiente, el desarrollo ulterior del análisis debe conducir a una definición más integral del desempeño que, al incluir la dimensión medioambiental, permita la determinación multidimensional del DES, facilitando además su medición y evaluación.

\subsection{La determinación multidimensional del DES: Una propuesta.}

La necesidad del enfoque multidimensional del desempeño empresarial se argumenta por la relación que existe entre la actividad económica de la empresa, los factores biofísicos del medio ambiente, el desarrollo humano de los trabajadores y el desarrollo social de la localidad donde la entidad está ubicada. Considérese que la economía, cuya esencia misma está en el proceso de producción, se sustenta en la actividad de intercambio del hombre con la naturaleza (Marx, 1980). Producir significa crear los bienes materiales y los servicios necesarios para la existencia y el desarrollo de la sociedad, y los factores de la producción son los medios de producción y la fuerza de trabajo.

La cantidad y calidad de materias primas (como objeto de trabajo), las características de los suelos, el tipo de clima, el agua y el aire, por citar algunos ejemplos, son factores biofísicos del medio ambiente que influyen en el nivel de productividad del trabajo de cualquier empresa. Por otro lado, el hombre, portador de la fuerza de trabajo, en tanto especie, es parte de la naturaleza y resultado de su estrecha conexión con el medio natural que lo rodea. Los factores biofísicos del medio ambiente condicionan el desarrollo de la producción empresarial, pues garantizan la provisión de recursos para los procesos productivos y la recepción de residuos, tanto de los recursos materiales (objetos y medios de trabajo), como de las condiciones necesarias para la reproducción de la fuerza de trabajo. A su vez, el crecimiento económico de las entidades posibilita la conservación del medio ambiente por medio del desempeño racional del individuo, condicionado a su vez por la educación medioambiental del hombre (sujeto de este proceso), cuando al transformar la naturaleza para su beneficio actúa en aras de conservarla. Esto expresa, a grandes rasgos, el vínculo entre las dimensiones económica, 
social y ambiental de la actividad empresarial, así como la necesidad de que todas sean igualmente priorizadas.

El crecimiento económico y el desarrollo social no son objetivos esencialmente antagónicos. En realidad son partes íntegras de un todo, y cada una de ellas se complementa entre sí. La madurez y la estabilidad del crecimiento económico de las empresas dependen en gran medida del nivel de desarrollo social que haya alcanzado la fuerza de trabajo; por otro lado, este está determinado por los ritmos de crecimiento económico, por el nivel de la producción, pues es en esta última esfera donde se crean los valores de uso indispensables para satisfacer las necesidades sociales y así se puede garantizar igualdad de condiciones para el despliegue de todas las potencialidades del individuo.

Téngase en cuenta que el hombre, factor decisivo del proceso de producción, podrá reproducir su capacidad de trabajar y aportar más en la medida en que pueda satisfacer con más holgura sus necesidades, si está físicamente más sano, si tiene una capacidad intelectual mayor, si cuenta con los conocimientos para utilizar tecnologías limpias y cumplir con la legislación en este orden, y si posee un alto nivel cultural y una elevada conciencia ambiental, es decir, si accede a un desarrollo multilateral.

Esto significa que, lejos de ser objetivos adversos, el desempeño económico de las empresas y el desarrollo social se complementan, y exigen tanto uno como el otro de un medio ambiente biofísico natural conservado, que a su vez será expresión del cumplimiento con lo ambientalmente legislado y con la utilización de tecnologías limpias.

Estas dimensiones deben ser entendidas como un sistema, o sea, como un conjunto de elementos interrelacionados entre sí, con un mutuo condicionamiento, de tal forma que las limitaciones de una de ellas inciden en el comportamiento del resto.

De esta forma, el DES se define por las autoras como: el cumplimiento cabal de las obligaciones inherentes a la empresa, en el orden ambiental, con el uso racional de los recursos naturales que utiliza; en lo económico, con la, maximización de los beneficios con inversiones ambientales en función de utilización de tecnologías limpias; desde lo legal, mediante la observancia de las leyes ambientales generales y específicas a su actividad; y desde el punto de vista social, con la minimización de los efectos negativos al entorno laboral y social.

Destáquese en dicha conceptualización la presencia de cuatro dimensiones del DES: 1) ambiental; 2) económico -tecnológica; 3) legal; y 4) social. Estas dimensiones representan las determinaciones cualitativas del DES. Y son las que posibilitan su enfoque multilateral y sintético, y permiten la exploración de sus determinaciones cuantitativas, su medición y la identificación de las variables de mayor influencia sobre él.Para una mejor comprensión de este enfoque, a continuación se detalla cada una de las dimensiones.

I. Dimensión ambiental: el desarrollo de una economía dependerá del sustento que su medio ambiente físico natural le proporcione. Es por ello que la empresa debe tener una conducta responsable hacia el entorno, y considerar las características específicas de su 
proceso productivo, es decir, los recursos naturales que constituyen las materias primas y materiales que utiliza como inputs ${ }^{4}$. También es importante el tratamiento adecuado de los residuales que genere dicho proceso y tener dominio sobre el ciclo de vida del producto. Así se contribuye a que la empresa actúe en correspondencia con las leyes objetivas que rigen en la naturaleza, que maneje de forma racional de los recursos que demanda, y que conozca que el flujo de extracción de los recursos renovables no puede ser mayor que el de su regeneración natural; la utilización de los recursos no renovables debe estar acompañada de la búsqueda de sustitutos y que el flujo de generación de residuos no debe estar por encima de la capacidad de carga del planeta para minimizar la contaminación. Esto propiciaría una conducta adecuada en términos de sostenibilidad, algo determinante si se retoma la interrelación hombre -medio ambiente naturalproducción para el desempeño económico.

II. Dimensión económico-tecnológica: la entidad alcanzará mayores niveles de eficiencia, eficacia y efectividad si es capaz de utilizar tecnologías limpias que le permitan avanzar en la excelencia y la competitividad empresarial, así como minimizar los costos asociados a la contaminación ambiental prevenible. Esto hará posible que el proceso de toma de decisiones evolucione en las empresas a favor de obtener un desempeño económico alto. La empresa debe lograr la maximización de los beneficios sobre la base de un consumo racional de los recursos, pero también a partir de la minimización de las externalidades ambientales negativas ${ }^{5}$ resultantes de la producción. Este dualismo de optimización obliga a que el desempeño, en el orden económico, sea preciso analizarlo a partir del comportamiento de variables tradicionales como la producción bruta o el valor agregado, la producción mercantil, pero mediante la incorporación del análisis sobre la utilización de tecnología limpia, o la dinámica de las inversiones ambientales y los costos de mitigación ambiental. La observancia estricta de la relación de los indicadores económicos y los ambientales contribuirá a obtener patrones de referencia para trazar y cumplir metas de desempeño económico sostenible.

III. Dimensión legal: La empresa debe disponer de toda la información referida a la legislación medioambiental vigente, tanto en términos generales, como aquellas regulaciones específicas de cada actividad productiva, para actuar a favor de su observancia. Lo anterior contribuirá a que la entidad minimice la actividad nociva de sus procesos para con el entorno laboral y social, y evite la inhabilitación de actividades

\footnotetext{
${ }^{4}$ Dominar a fondo las características de los recursos naturales que emplea permite a una empresa no solo clasificarlos en recursos renovables o no renovables, sino además conocer qué tratamiento darles, si son empleados eficientemente, o si alguno de ellos puede ser reutilizado, reciclado, recuperado o tratado.

${ }^{5}$ Considérese que las externalidades negativas de la producción son aquellos efectos indeseados que la actividad productiva provoca imponiendo costos adicionales a la sociedad y que no se reflejan en los precios de mercado, y que se constituyen en fuentes de ineficiencia económica.
} 
industriales, con el consiguiente costo asociado a los cierres temporales. Incorporar la materia legislativa a la cultura empresarial no solo impedirá contravenir la legislación ambiental, sino que ayudará a que las cuotas abonadas no se traduzcan en una reducción de los beneficios de las entidades al cierre de sus operaciones contables. Es de esperar entonces que sean mínimas las acciones de saneamiento, que siempre originan mayores costos que los asociados a las actividades preventivas.

IV. Dimensión social: A partir de las características del proceso productivo la empresa debe asumir y cumplir su responsabilidad social, para lo cual debe evaluar la afectación que pueden causar a la salud la presencia de agentes ambientales procedentes del proceso de producción. Esto favorecerá que el individuo, en la empresa, concientice la necesidad e importancia de actuar en aras de proteger su salud, a partir de un entorno laboral y local sano. Además, es necesario considerar las afectaciones que puede provocar la empresa en la localidad donde esté situada, para que se sensibilice al respecto, y su accionar esté encaminado a minimizar el impacto negativo que su actividad productiva ocasione. Por tanto, hay que tener en cuenta las externalidades negativas y minimizarlas. De ahí la importancia de establecer la diferencia entre costo empresarial y costo social, pues la producción óptima de una empresa puede no ser la óptima para la sociedad. La explicación radica en el hecho de que una producción que afecte negativamente a la población circundante, e incluso, a los trabajadores empleados en ella, mientras más crezca más daño provoca. Por ello, el punto óptimo de producción para la sociedad está allí donde el costo marginal social es igual al precio del producto. Este costo marginal social es la sumatoria del costo marginal empresarial y del costo marginal externo. Es significativo medir los costos sociales y establecer el umbral de las llamadas deseconomías externas, es decir, a partir de qué nivel de producción, los costos sociales son mayores que los costos empresariales, con lo cual, aún cuando sea rentable para la empresa incrementar sus volúmenes de bienes y/o servicios, no lo es para la sociedad y no debe hacerlo.

En la literatura microeconómica no existe un consenso acerca de cómo medir el costo marginal externo. A los efectos de esta investigación los costos marginales externos pueden determinarse por las erogaciones que realiza el gobierno en programas de saneamiento ambiental, por las enfermedades profesionales y por las violaciones de las normas e higiene del trabajo que, en condiciones de una cobertura médica gratuita, provoca costos sociales enormes. Todo lo anterior permitirá que la empresa cumpla las normas de protección e higiene del trabajo, momento importante en su desempeño social y así asegurará las condiciones para preservar la salud y la reproducción del individuo, portador de la fuerza de trabajo y ser social, conservando, a su vez, el entorno local donde la entidad esté enclavada.

Lo anteriormente expuesto demuestra la importancia de que las empresas alcancen un desempeño sostenible $y$ que este se fundamente en un enfoque multidimensional, que exige, a su vez, una rigurosa información para que el individuo y, 
en especial, los decisores de política, ganen en conocimientos sobre cada una de estas dimensiones, y así se logre sensibilizarlos y comprometerlos para actuar en esta dirección.

Ello pone de manifiesto uno de los elementos más significativos del análisis multidimensional del DES: la importancia vital que desempeña la educación medioambiental empresarial en la búsqueda de escenarios promisorios.

Resulta complejo lograr un DES sin comprender, desde lo cognitivo, la interrelación entre la empresa y su entorno (físico-natural, económico-tecnológico, legal y social), sin sensibilizar al individuo con la crisis ambiental contemporánea, sin lograr cambiar sus esquemas de pensamiento y patrones de conducta. Avanzar en términos de sostenibilidad es imposible sin la educación medioambiental empresarial como elemento integrante de la cultura empresarial, asociado en lo fundamental a los procesos de capacitación de los recursos humanos. Lamentablemente, este importante aspecto es ignorado por algunos decisores de política.

\section{CONCLUSIONES}

- Es necesario el enfoque multidimensional del desempeño empresarial sostenible para lograr un análisis más integral de la relación de la empresa con el entorno ambiental, económico social y legal en que realiza sus actividades.

- EI DES tiene cuatro dimensiones: ambiental; económico -tecnológica; legal; y social. Estas dimensiones representan sus determinaciones cualitativas, que hacen posible su enfoque multilateral y sintético, y que permiten la exploración de sus determinaciones cuantitativas, su medición y la identificación de las variables de mayor influencia sobre el que mismo.

- La influencia activa de la educación medioambiental en todas las dimensiones del desempeño empresarial la convierte en una herramienta fundamental para avanzar en la senda de la sostenibilidad en las entidades.

\section{BIBLIOGRAFÍA}

Bueno Campos, E. 1994. Economía de Empresas (Análisis de las Decisiones Empresariales), Editorial EMPES.

Bueno Campos, E. 1989. Dirección Estratégica de la Empresa. Metodología, Técnicas y Casos, Editorial Pirámide S.A., Madrid, España.

BUFFA E. S. (1991), Administración de la Producción y las Operaciones, Editorial Limusa, México.

Camisón Zornoza, C. 2007. “Estrategias competitivas y desempeño empresarial: estudio comparativo". Revista Investigaciones Europeas de Dirección y Economía de la Empresa, Vol. 13, № 3, pp. 161-182. Recuperado el 18 de noviembre de 2010 de http:// www.aedem-virtual.com 
Cantero Cora, H. 2011. "Procedimiento para el mejoramiento de la evaluación del desempeño empresarial a partir de un enfoque basado en procesos. Caso de estudio ETECSA". Tesis de Maestría. Departamento de Ingeniería Industrial. Universidad de Holguín.

Cantero, H. 2010. “Apuntes acerca del desempeño empresarial”. Revista Observatorio de la Economía Latinoamericana, № 141. Recuperado el 25 de junio de 2011 de http:// www.eumed.net

Castell, M. 2008. “Globalización, tecnología, trabajo, empleo y empresa”. Revista "La Factoría", № No. 7, España. Recuperado el 23 de junio de 2012 de http://www.lafactoriaweb.com.

Coase, H.R. 1960. "El problema del Costo social". Estudios Públicos № 45,1992, pp 81134.

Coma Rodríguez, R y Nogueira Rivera, D. 2010. “Procedimiento para el desarrollo de un cuadro de mando integral. Caso de estudio en la empresa de suministros y transporte agropecuario de Santi Spíritus". Revista Observatorio de la Economía Latinoamericana, № 134. Recuperado el 4 de mayo de 2011 de http:// www.eumed.net

Decreto Ley 187. Bases Generales del Perfeccionamiento Empresarial. 1998, Gaceta Oficial de La República de Cuba. La Habana.

Decreto Ley No 252. Sobre la continuidad y el fortalecimiento del sistema de dirección y gestión empresarial cubano. 2007. Gaceta Oficial de la República de Cuba, La Habana.

Decreto Ley No 281. Reglamento para la implementación y consolidación del sistema de dirección y gestión empresarial estatal. 2007.Gaceta Oficial de la República de Cuba, La Habana.

Drucker, P. 1964.Managing for results, Editorial Harper \& Row, Nueva York.

Durán Romero, G. 2007. Empresa y Medio Ambiente. Políticas de Gestión Ambiental, Ediciones Pirámides Grupo Anaya S.A.. Madrid, España.

Espinosa Mané, S. 2011. "Evaluación del desempeño de la empresa estatal socialista cubana en la nueva etapa". Revista Cubana de Gestión Empresarial. 7, 2.

Estopiñan Marrero, A. 2010. Metodología para la Evaluación del Desempeño Empresarial a partir de la Organización de los Procesos. Aplicación en el Telepunto de Holguín. Tesis de grado, Departamento de Ingeniería Industrial. Universidad de Holguín.

Garmendía Salvador, A. 2005. Evaluación del Impacto Ambiental, Editorial Pearson Educación, Madrid, España.

Guía de Formación de Desarrollo Sostenible Empresarial. 2005. Recuperado el 2 de mayo de 2011 de http://www.endesa.cl/pdf20056

Informe de Sostenibilidad Endesa Chile 2005. Recuperado el 4 de mayo de 2011 de http:// www.endesa.cl

Kaplan, R.S. y Norton, D.P. 1996. "Using the Balanced Scorecard as a Strategic Management System". Harvard Business Review, January-February, pp75-85.

Koontz, H. et al. 1975. Curso de administración moderna. Un análisis de las Funciones de la Administración, cuarta edición, Edición Revolucionaria, Universidad de la Habana, La Habana, Cuba.

Koontz, H. et al. 1994. Administración, una perspectiva global, décima edición, México. 
Ley № 81 del Medio Ambiente. 1997. Gaceta Oficial de la República de Cuba. La Habana. Leyva Cardeñosa, E. 2002. Procedimiento metodológico para la evaluación del desempeño empresarial a partir de la organización de los procesos. Aplicación en la Villa el Bosque, Tesis presentada en opción al título académico de máster. Universidad de Camagüey.

Leyva Rodríguez, M. 2002. "Evaluación del desempeño empresarial a partir de la organización del proceso en la empresa de Gases Industriales". Revista Trimestral, año VIII, № 3. Recuperado el 23 de septiembre de 2011 en http://www.ciencias.holguin.cu

Lugones, G. y Suaréz, Diana. 2007. "Conducta innovativa y desempeño empresarial". Recuperado el 23 de noviembre de 2010 de http:// www.centroredes.org.ar

Marc Chordá, I. 2009. "Desempeño en empresas de economía social. Un modelo para su medición". CIRIEC-España. Revista de Economía Pública, Social y Cooperativa, № 64, pp105- 131. Recuperado el 20 de diciembre de 2010 de http:// www.redalyc.uaemex.mx

Martínez Rivadeneira, R. 2005. "Balanced Scorecard Financiero". Recuperado el 20 de diciembre de 2010 de http:// www.sfcaltagerencia.com

Marx, K. (ed.1980) El Capital, Tomo I, Editorial de Ciencias Sociales, Ciudad de La Habana.

Marx, K. y Engels, F. (ed.1971). Obras Escogidas en tres tomos, Tomo I, Editorial progreso, Moscú.

Mayo Alegre, J.C., Laredo Carballo, N. y Reyes Benítez, S. 2009. "Procedimiento para evaluar la eficacia organizacional", Revista Contribuciones a la Economía, Recuperado el 11 de noviembre de 2011 de http://www.eumed.net

Medina León, A. 2010. "Relevancia de la Gestión por Procesos en la Planificación Estratégica y la Mejora Continua". Revista Eídos № 2.

Mesa, E. 2007. "La evaluación del desempeño: herramienta vital en la dirección organizacional", en Observatorio de la Economía Latinoamericana № 73. Recuperado el 4 de mayo de 2011 de http:// www.eumed.net

Norma Cubana. ISO 14001. 2007. Sistema de gestión Ambiental. Recuperado el 2 de setiembre de 2011 de http:// e www.iso.ch

Norma de Aseguramiento de Sostenibilidad. 2008. Recuperado el 14 de enero de 2010 de http:// www.accountability.org

Norma Internacional ISO 14031. 1999. Environmental management - Environmental performance evaluation - Guidelines. Recuperado el 4 de mayo de 2011 de http:// www.iso.org/iso/catalogue_detail.htm\%

Núnez, G. 2003. La responsabilidad social corporativa de desarrollo sostenible", Naciones Unidas, Santiago de Chile.

Pérez Bengochea, V. 2008. "Desempeño Empresarial”. Recuperado el 12 de marzo de 2010 de http:// www.degerencia.com

Porter, M. 1990. Ventaja Competitiva. Editorial Continental S.A., México.

Silveira Pérez, Y. 2010. "Benchmarking, gestionando el desempeño empresarial a través de las mejores prácticas." en Observatorio de la Economía Latinoamericana № 131. Recuperado el 11 de noviembre de 2011 de http://www.eumed.net

Stoner, J. 1996. Administración. Sexta edición, Prentice-Hall, Inc., México. 